Mathematical Research Letters 1, 597-600 (1994)

\title{
PERIODIC BUNCHING AND INVARIANT FOLIATIONS
}

\author{
Boris HASSELBLATT
}

\begin{abstract}
A вstract. Control of contraction and expansion rates on periodic orbits of a hyperbolic dynamical system gives analogous control on all orbits. This leads to a sharp condition for the regularity of the invariant foliations in terms of bunching at periodic points.
\end{abstract}

This paper shows that "bunching" information, which is relevant to various questions about regularity of invariant structures of hyperbolic systems, is transferred from periodic orbits to nonperiodic ones. A motivation is the result of $[\mathrm{Ha}]$ which says that uniform $\alpha$-bunching of all orbits of an Anosov system implies that the Anosov splitting is $C^{\alpha},(\alpha \in(0,2))$, whereas the failure of any periodic orbit to be $\alpha$-bunched will cause (for a generic set of symplectic Anosov systems) the Anosov splitting not to be $C^{\alpha}$. The present paper (negatively) answers the obvious question of whether it is possible to have all periodic orbits $\alpha$-bunched without having all orbits uniformly bunched: If all periodic orbits of a topologically transitive Anosov flow (or diffeomorphism) are bunched then all orbits are uniformly bunched.

A consequence is, using [Ha], a completely sharp description, in terms of periodic points only, of what bunching information is needed to obtain a given regularity of the Anosov splitting (Corollaries 1 and 2). It should be emphasized that the main result, although stated for Anosov flows, holds also for Anosov diffeomorphisms as well as compact locally maximal hyperbolic sets for flows or local diffeomorphisms.

This paper is based on a paper of $\mathrm{U}$. Hamenstädt $[\mathrm{H}]$ where an analogous result is shown for "absolute bunching". Extensive background is provided by $[\mathrm{Ha}]$.

1991 Mathematics Subject Classification. Primary 58F15, 58F17, 58F18, Secondary 53C12, 53C20, 53C35.

Received June 14, 1994. 
Definition 1. A flow $\Phi^{t}$ on a compact Riemannian manifold $M$ is called Anosov with Anosov splitting $\left(E^{u}, E^{s}\right):=\left(E^{s u} \oplus E^{\Phi}, E^{s s} \oplus E^{\Phi}\right)$ if $T M=$ $E^{s u} \oplus E^{s s} \oplus E^{\Phi}, E^{\Phi}=\operatorname{span}\{\dot{\Phi}\} \neq\{0\}$ and there exist $\lambda<1, C>0$ such that for all $p \in M, t>0$

$$
\begin{aligned}
& \left\|D \Phi^{t}(v)\right\| \leq C \lambda^{t}\|v\| \quad \text { for } v \in E^{s s}(p) \quad \text { and } \\
& \left\|D \Phi^{-t}(u)\right\| \leq C \lambda^{-t}\|u\| \quad \text { for } u \in E^{s u}(p) \text {. }
\end{aligned}
$$

For an Anosov flow $\Phi^{t}$ and $t>0$ define

$$
\begin{aligned}
Q_{t}^{1}(x) & :=\frac{1}{t} \sup \left\{\log \left(\frac{\left|d \Phi^{t}(X)\right|}{|X|}\right) \mid 0 \neq X \in E_{x}^{s s}\right\} \\
Q_{t}^{2}(x) & :=\frac{1}{t} \sup \left\{\log \left(\frac{\left|d \Phi^{-t}(X)\right|}{|X|}\right) \mid 0 \neq X \in E_{x}^{s u}\right\} \\
Q_{t}^{3}(x) & :=\frac{1}{t} \inf \left\{\log \left(\frac{\left|d \Phi^{t}(X)\right|}{|X|}\right) \mid 0 \neq X \in E_{x}^{s s}\right\} .
\end{aligned}
$$

Note that these are all negative and bounded independently of $t>0$. We say that $\Phi^{t}$ is $\alpha$-u-bunched if there exists a $t>0$ such that

$$
\sup _{x \in M}\left(Q_{t}^{1}(x)+Q_{t}^{2}(x)-\alpha Q_{t}^{3}(x)\right)<0,
$$

$\alpha$-s-bunched if $\Phi^{-t}$ is $\alpha$-u-bunched, $\alpha$-bunched if $\Phi^{t}$ and $\Phi^{-t}$ are $\alpha$-ubunched. $\alpha$-u-bunching yields $C^{\alpha+\varepsilon}$ unstable distributions and $\alpha$-s-bunching yields $C^{\alpha+\varepsilon}$ stable distributions [Ha]. Here $\alpha$ is arbitrary, but if $\Phi^{t}$ is $\alpha$-bunched then necessarily $\alpha<2$ [Ha]. For symplectic systems $\alpha$-ubunching and $\alpha$-s-bunching are equivalent.

We chose this definition for convenience, but it is easily seen to be equivalent to that in [Ha]. The purpose of this paper is to show that bunching information can be obtained from information about periodic points only:

Definition 2. We say that the periodic orbits of an Anosov flow $\Phi^{t}$ are $\alpha$-u-bunched if for every periodic point $p$ of period $\tau$ the absolute values of the eigenvalues of $d \Phi^{\tau}\left\lceil_{E_{p}^{s s}}\right.$ are contained in an interval $\left[q_{3}^{p}, q_{1}^{p}\right]$ and the absolute values of the eigenvalues of $d \Phi^{\tau} \uparrow_{E_{p}^{s s}}$ are bounded by $q_{2}^{p}$, where $\log q_{1}^{p}+\log q_{2}^{p}-\alpha \log q_{3}^{p} \leq 0$. The periodic orbits are $\alpha$-bunched if they are $\alpha$-u-bunched under both $\Phi^{t}$ and $\Phi^{-t}$.

THEOREM. If $\Phi^{t}$ is a transitive Anosov flow on a compact manifold $M$ whose periodic orbits are $\alpha$-u-bunched, then $\Phi^{t}$ is $(\alpha-\varepsilon)$-u-bunched.

This completes the investigations in [Ha] (see Proposition 1.16 there) by giving, together with the results in $[\mathrm{Ha}]$, the following precise descriptions 
of what bunching information is needed for any given regularity of the Anosov splitting. In both Corollaries the first sentence follows from the Theorem and the second from $[\mathrm{Ha}]$.

Corollary 1. All transitive Anosov flows whose periodic orbits are $\alpha$-ubunched have $C^{\alpha-\varepsilon}$ unstable distribution. Conversely, among the symplectic Anosov flows whose periodic orbits are not all $\alpha$-u-bunched there is an open dense (in any $C^{k}$ topology) set of flows for which neither stable nor unstable distribution is $C^{\alpha-\varepsilon}$.

Corollary 2. A negatively curved Riemannian metric all of whose closed geodesics are $\alpha$-bunched has $C^{\alpha-\varepsilon}$ horospheric foliations. On the other hand, the set of negatively curved Riemannian metrics on a manifold which have a closed, not $\alpha$-bunched, geodesic through a point whose injectivity radius exceeds $(\log 2) / K$, where $-K^{2}$ is the maximal sectional curvature, contains an open dense set of metrics whose horospheric foliations are not $C^{\alpha-\varepsilon}$.

Proof. Let $\mathcal{M}$ denote the space of $\Phi^{t}$-invariant Borel probability measures on $M, F_{k, \epsilon}(x):=\max \left(0, Q_{2^{k}}^{1}+Q_{2^{k}}^{2}-(\alpha-\epsilon) Q_{2^{k}}^{3}\right)$. The proof of Lemma 1 of $[\mathrm{H}]$ shows

Lemma 1. For $\epsilon, \delta>0$ there is a $k(\epsilon, \delta)$ with $\int F_{k, \epsilon} d \eta<\delta$ for $\eta \in \mathcal{M}$, $k \geq k(\epsilon, \delta)$.

Take $r(\epsilon) \in \mathbb{N}$ such that $e^{-a \epsilon /(8+4 \alpha)}|X| \leq\left|d \Phi^{t}(X)\right| \leq e^{a \epsilon /(8+4 \alpha)}|X|$ for $|t| \leq 1 / r(\epsilon), a:=-\sup _{x \in M, t>0} Q_{t}^{3}(x)>0$. Let $\varkappa(\epsilon, \delta):=k(\epsilon / 4, a \epsilon \delta / 4 r)$, $\tau(\epsilon, \delta):=2^{\varkappa(\epsilon, \delta)}$ and $\chi_{\epsilon, \delta}$ the characteristic function of

$$
A(\epsilon, \delta):=\left\{x \in M \mid Q_{\tau(\epsilon, \delta)}^{1}(x)+Q_{\tau(\epsilon, \delta)}^{2}(x)-(\alpha-\epsilon) Q_{\tau(\epsilon, \delta)}^{3}(x)<0\right\} .
$$

Lemma 2. For $\epsilon, \delta>0$ there exists $l(\epsilon, \delta) \in \mathbb{N}$ such that for $x \in M$ and $l \geq l(\epsilon, \delta)$

$$
\frac{1}{l} \sum_{i=0}^{l-1} \chi_{\epsilon, \delta}\left(\Phi^{\tau(\epsilon, \delta)}(x)\right)>1-\delta
$$

Proof. Let $\tau=\tau(\epsilon, \delta), A_{0}:=\left\{x \in M \mid Q_{\tau}^{1}(x)+Q_{\tau}^{2}(x)-\left(\alpha-\frac{\epsilon}{2}\right) Q_{\tau}^{3}(x)<\right.$ $0\}, \chi=\chi_{\epsilon, \delta}, A=A(\epsilon, \delta), r=r(\epsilon), \chi_{0}$ the characteristic function of $A_{0}$ and $\eta_{l}(x)$ the measure defined by $\int \varphi d \eta_{l}(x)=(1 / \tau l) \int_{0}^{\tau l} \varphi\left(\Phi^{t}(x)\right) d t$. There exists an $l_{0} \in \mathbb{N}$ such that $\int \chi_{0} d \eta_{l}(x)>1-\delta / r$ for $l>l_{0}$ and $x \in M$ because otherwise $\int F_{\varkappa, \epsilon / 4} d \eta_{l}(x) \geq-(\delta / r)\left(Q_{\tau}^{3} \epsilon / 4\right) \geq a \epsilon \delta / 4 r$ and 
$\int F_{\varkappa, \epsilon / 4} d \eta \geq a \epsilon \delta / 4 r$ for any ( $\Phi^{t}$-invariant) weak*-accumulation point $\eta$ of $\left\{\eta_{l}(x)\right\}_{l \in \mathbb{N}, x \in M}$, contrary to the choice of $\varkappa$.

Next observe, similarly to $[\mathrm{H}]$, that for $x \in A_{0}$ and $|t| \leq \tau / r$ by choice of $r$

$$
\begin{aligned}
Q_{\tau}^{1}\left(\Phi^{t}(x)\right)+Q_{\tau}^{2}\left(\Phi^{t}(x)\right)-(\alpha-\epsilon) Q_{\tau}^{3}\left(\Phi^{t}(x)\right) \\
\leq Q_{\tau}^{1}(x)+\frac{a \epsilon}{4+2 \alpha}+Q_{\tau}^{2}(x)+\frac{a \epsilon}{4+2 \alpha}-(\alpha-\epsilon)\left(Q_{\tau}^{3}(x)+\frac{a \epsilon}{4+2 \alpha}\right) \\
=Q_{\tau}^{1}(x)+Q_{\tau}^{2}(x)-\left(\alpha-\frac{\epsilon}{2}\right) Q_{\tau}^{3}(x)+\left[\frac{\epsilon}{2} Q_{\tau}^{3}(x)+(2+\alpha-\epsilon) \frac{a \epsilon}{4+2 \alpha}\right] \\
\quad<Q_{\tau}^{1}(x)+Q_{\tau}^{2}(x)-\left(\alpha-\frac{\epsilon}{2}\right) Q_{\tau}^{3}(x) .
\end{aligned}
$$

Thus $x \notin A$ implies $\Phi^{t}(x) \notin A_{0}$ for $|t| \leq \tau / r$ and (1) holds for $x \in M$ and $l>l_{0}$ because otherwise

$$
\frac{1}{\tau l} \int_{0}^{\tau l} 1-\chi_{0}\left(\Phi^{t}(x)\right) d t \geq \frac{1}{\tau} \delta \tau / r=\frac{\delta}{r} \text { and } \frac{1}{\tau l} \int_{0}^{\tau l} \chi_{0}\left(\Phi^{t}(x)\right) d t \leq 1-\frac{\delta}{r},
$$

contrary to the choice of $l_{0}$.

Let $C:=\epsilon+\sup _{x \in M, t>0}\left(Q_{t}^{1}(x)+Q_{t}^{2}(x)-(\alpha-\epsilon) Q_{t}^{3}(x)\right), \delta=1 /((2 C / a \epsilon)+$ $1)$ and $l:=l(\epsilon / 2, \delta), \tau:=\tau(\epsilon / 2, \delta)$. To show $\left(*_{(\alpha-\epsilon)}\right)$ for $t=l \tau$ note that

$$
Q_{\tau}^{1}\left(\Phi^{\tau i}(x)\right)+Q_{\tau}^{2}\left(\Phi^{\tau i}(x)\right)-\left(\alpha-\frac{\epsilon}{2}\right) Q_{\tau}^{3}\left(\Phi^{\tau i}(x)\right)<0
$$

if $\chi_{\epsilon / 2, \delta}\left(\Phi^{\tau i}(x)\right)=1$ and

$$
Q_{\tau}^{1}\left(\Phi^{\tau i}(x)\right)+Q_{\tau}^{2}\left(\Phi^{\tau i}(x)\right)-(\alpha-\epsilon) Q_{\tau}^{3}\left(\Phi^{\tau i}(x)\right) \leq C-\epsilon
$$

always, so that

$$
\begin{aligned}
Q_{t}^{1}(x)+Q_{t}^{2}(x)-(\alpha-\epsilon) & Q_{t}^{3}(x) \\
& \leq \sum_{i=0}^{l-1} Q_{\tau}^{1}\left(\Phi^{\tau i}(x)\right)+Q_{\tau}^{2}\left(\Phi^{\tau i}(x)\right)-(\alpha-\epsilon) Q_{\tau}^{3}\left(\Phi^{\tau i}(x)\right) \\
& <\frac{-a \epsilon}{2}(1-\delta)+(C-\epsilon) \delta=-\epsilon \delta . \quad \square
\end{aligned}
$$

\section{References}

[H] Ursula Hamenstädt, Anosov flows which are uniformly expanding at periodic points, Ergodic Theory and Dynamical Systems 14 (1994), 299-304.

[Ha] Boris Hasselblatt, Regularity of the Anosov splitting and of horospheric foliations, Ergodic Theory and Dynamical Systems (to appear).

Department of Mathematics, Tufts University, Medford, M A 02155-5597. E-mail address: BHasselb@Jade.Tufts.edu 